\title{
PENELOPE-2008: A Code System for Monte Carlo Simulation of Electron and Photon Transport
}

\author{
Workshop Proceedings \\ Barcelona, Spain \\ 30 June-3 July 2008
}
Francesc Salvat, José M. Fernández-Varea, Josep Sempau Facultat de Física (ECM)
Universitat de Barcelona Spain

\author{
(C) OECD 2009 \\ NEA No. 6416 \\ NUCLEAR ENERGY AGENCY \\ ORGANISATION FOR ECONOMIC CO-OPERATION AND DEVELOPMENT
}

